\title{
Accuracy of an MR-only workflow for prostate radiotherapy using semi-automatically burned-in fiducial markers
}

\author{
Karin Goudschaal ${ }^{*}$ (D, F. Beeksma, M. Boon, M. Bijveld, J. Visser, K. Hinnen and Z. van Kesteren
}

\begin{abstract}
Background: The benefit of MR-only workflow compared to current CT-based workflow for prostate radiotherapy is reduction of systematic errors in the radiotherapy chain by 2-3 $\mathrm{mm}$. Nowadays, MRI is used for target delineation while CT is needed for position verification. In MR-only workflows, MRI based synthetic CT (sCT) replaces CT. Intraprostatic fiducial markers (FMs) are used as a surrogate for the position of the prostate improving targeting. However, FMs are not visible on sCT. Therefore, a semi-automatic method for burning-in FMs on SCT was developed. Accuracy of MR-only workflow using semi-automatically burned-in FMs was assessed and compared to CT/MR workflow.

Methods: Thirty-one prostate cancer patients receiving radiotherapy, underwent an additional MR sequence (mDIXON) to create an sCT for MR-only workflow simulation. Three sources of accuracy in the CT/MR-and MR-only workflow were investigated. To compare image registrations for target delineation, the inter-observer error (IOE) of FM-based CT-to-MR image registrations and soft-tissue-based MR-to-MR image registrations were determined on twenty patients. Secondly, the inter-observer variation of the resulting FM positions was determined on twenty patients. Thirdly, on 26 patients CBCTs were retrospectively registered on SCT with burned-in FMs and compared to CT-CBCT registrations.

Results: Image registration for target delineation shows a three times smaller IOE for MR-only workflow compared to CT/MR workflow. All observers agreed in correctly identifying all FMs for 18 out of 20 patients (90\%). The IOE in CC direction of the center of mass (COM) position of the markers was within the CT slice thickness ( $2.5 \mathrm{~mm}$ ), the IOE in AP and RL direction were below $1.0 \mathrm{~mm}$ and $1.5 \mathrm{~mm}$, respectively. Registrations for IGRT position verification in MR-only workflow compared to CT/MR workflow were equivalent in RL-, CC- and AP-direction, except for a significant difference for random error in rotation.
\end{abstract}

Conclusions: MR-only workflow using SCT with burned-in FMs is an improvement compared to the current CT/ MR workflow, with a three times smaller inter observer error in CT-MR registration and comparable CBCT registration results between $\mathrm{CT}$ and $\mathrm{SCT}$ reference scans.

Trial registry Medical Research Involving Human Subjects Act (WMO) does apply to this study and was approved by the Medical Ethics review Committee of the Academic Medical Center. Registration number: NL65414.018.18. Date of registration: 21-08-2018.

Keywords: IGRT, Prostate cancer, CT/MR conventional workflow, MRI, MR-only workflow, Synthetic CT, Semiautomatic FM identification, Burning-in gold fiducial markers

*Correspondence: k.n.goudschaal@amsterdamumc.nl

Department of Radiation Oncology, University of Amsterdam, Amsterdam

UMC, Meibergdreef 9, 1105AZ Amsterdam, The Netherlands original author(s) and the source, provide a link to the Creative Commons licence, and indicate if changes were made. The images or other third party material in this article are included in the article's Creative Commons licence, unless indicated otherwise in a credit line to the material. If material is not included in the article's Creative Commons licence and your intended use is not permitted by statutory regulation or exceeds the permitted use, you will need to obtain permission directly from the copyright holder. To view a copy of this licence, visit http://creativecommons.org/licenses/by/4.0/. The Creative Commons Public Domain Dedication waiver (http://creativeco mmons.org/publicdomain/zero/1.0/) applies to the data made available in this article, unless otherwise stated in a credit line to the data. 


\section{Introduction}

External beam radiotherapy (EBRT) has been an established treatment for low to high-risk prostate cancer for decades $[1,2]$. A CT scan is conventionally used for radiation treatment planning (RTP) and for image-guidance during treatment (IGRT). In recent years, MRI has become an integral part of prostate radiotherapy for its excellent soft tissue contrast, improving prostatic cancer detection and localization significantly [3-6]. MRI is therefore preferred to $\mathrm{CT}$ for prostate delineation $[1,7]$ and has been incorporated into the pre-treatment process $[1,6,8-10]$ by means of a registration of the MRI with the CT used for RTP. However, CT/MRI registration is only accurate up to $2-3 \mathrm{~mm}$ for prostate cancer patients, and therefore introduces a systematic error in the radiotherapy chain [11-13]. This systematic error can be mitigated by using only MR images and omitting the CT scan [14]. Differences in anatomy, which inevitably occur when using both a CT and an MRI scan, is less of a problem by using an MRI scan only for delineation. Also, the patient does not have to undergo a CT examination, which reduces the radiation burden.

Nowadays commercial software is available to create a synthetic Computed Tomography ( $\mathrm{sCT}$ ) based on MRI that can be used for RTP and as a reference image for IGRT [10]. The workflow using a sCT instead of a $\mathrm{CT}$ is hereafter referred to as the MR-only workflow, in contrast to what is referred to as a CT/MR workflow, for which MR is registered with a CT that is used in the conventional manner.

There are roughly three available IGRT options in the EBRT workflow for prostate cancer patients using linear accelerators equipped with $\mathrm{kV} / \mathrm{MV}$ imaging [3]: registration using the bony anatomy of the small pelvic area, the soft tissue structures (e.g. prostate gland), or with the aid of fiducial markers (FMs) implanted within the prostate prior to imaging. Registration on bony anatomy alone goes hand in hand with a large PTV margin to cover for the substantial prostate motion relative to the pelvic bony anatomy $[15,16]$. Registration on soft tissue structures such as the prostate gland is challenging because the prostate gland can hardly be distinguished from adjacent structures, especially when the soft tissue contrast of $\mathrm{kV} /$ MV imaging is reduced by moving gas-induced artefacts $[17,18]$. Registration using FM leads to minimal intraobserver error as intraprostatic FMs are a surrogate for the position of the prostate $[19,20]$. Performing IGRT with FMs significantly improves prostate targeting, and therefore smaller PTV margins are required, with the benefit of better OAR sparing [7, 16-18, 20-23].

Using FMs for position verification is not straightforward in the MR-only workflow as the currently available commercial software to create a $\mathrm{sCT}$ is not yet able to visualize the FMs in the sCT with high reliability [7, 20]. As a workaround, a semi-automatic method can be used to identify and burn-in the FMs on the sCT $[7,20]$. A (semi)-automatic method to overcome any missed FM identification on MRI has been developed by several research groups [2, 7, 20, 24, 25].

An MR-only workflow with IGRT based on burnedin FMs for prostate radiotherapy has been investigated in a number of clinics $[1,2,20,24,26]$ and successfully introduced [24, 26]. However, the existing studies did not compare the systematic errors in the radiotherapy chain of the MR-only workflow to the conventional CT/MR workflow. Furthermore, to the best of our knowledge, the studies regarding IGRT with CBCT based on burned-in FMs were either obtained with translations only, instead of translations and rotations, or obtained with manual FM-based registration, instead of using an automatic matching algorithm [27].

The purpose of this study was to assess the accuracy of a simulated MR-only workflow using a sCT with semiautomatically burned-in gold FMs and compared it to the conventional CT/MR workflow. Three sources of inaccuracy were evaluated: the inter-observer variation of the image registration for target delineation, the interobserver variation of burned-in FM positions and the accuracy of image registration for position verification with CBCT using burned-in FMs.

\section{Methods \\ Study population}

Patients receiving curative radiotherapy for prostate cancer at our institute between October 2018 and May 2019 were asked to participate in this study. In accordance with the regulations of the local ethics committee, 31 patients gave their informed consent to add an MRI sequence for generating a $\mathrm{SCT}$ to the standard clinical MRI acquisitions used for target delineation.

Patients with a 3T MRI contra indication were excluded, as well as patients with hip prostheses or with an abdominal diameter exceeding $50 \mathrm{~cm}$ in left-right or $30 \mathrm{~cm}$ in anterior-posterior direction, since a sCT could not be generated for these cases.

\section{Current clinical CT/MR workflow}

Following the current clinical workflow (see Fig. 1, CT/ MR workflow in blue), four cylindrical gold FMs (diameter $1 \mathrm{~mm}$, length $5 \mathrm{~mm}$, RT-Idea B.V.) were implanted in the patient's prostate, at least four days prior to CT- and MR imaging. To minimize anatomical changes between the scans, the time between $\mathrm{CT}$ and MR scans was less than two hours.

The planning $\mathrm{CT}$ was acquired with an in-plane resolution of $1 \times 1 \mathrm{~mm}^{2}$ and a $2.5 \mathrm{~mm}$ slice thickness 


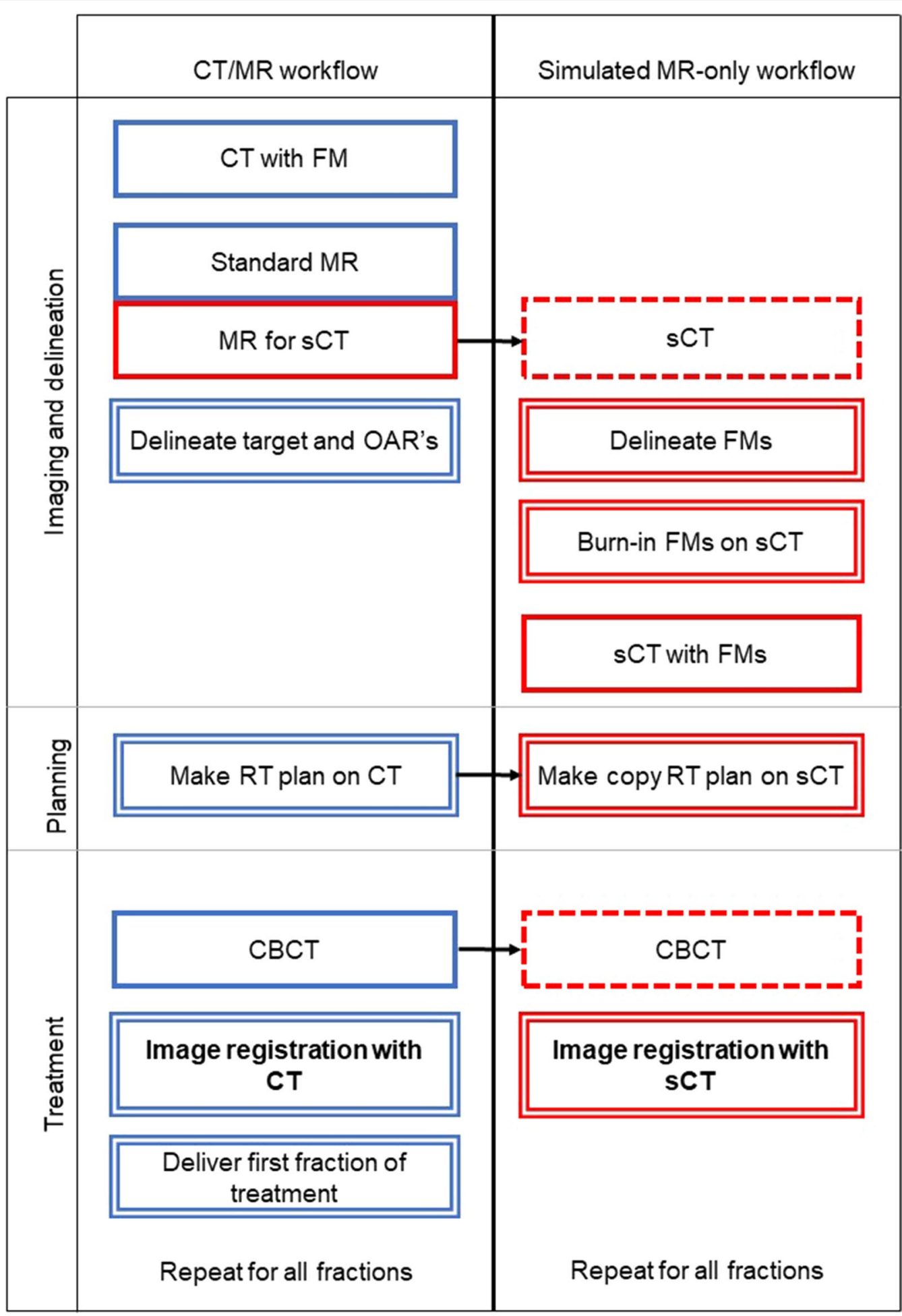

Fig. 1 Flowchart CT/MR workflow (in blue) and simulated MR-only workflow (in red). Thick line: image acquisition; double line: procedure step; dotted line: copied image; thick printed text: outcome used for evaluation 
(LightSpeed RT16 CT, GE). The patients were instructed to have a filled bladder for both the CT examination and each radiotherapy fraction, which makes it possible to spare the bladder during treatment. To achieve this, patients were asked to empty their bladder and to subsequently drink $500 \mathrm{ml}$ of water $1.5 \mathrm{~h}$ before the appointment.

The MRI was acquired in treatment position (Ingenia 3 T MRI with RT Oncology configuration, Philips Healthcare, Best, The Netherlands). The patients were not instructed to have a filled bladder for the MRI examination and were allowed to urinate before the MRI examination, if necessary. The standard clinical acquisition protocol consisted of transversal and sagittal T2-weighted images (which are referred to as T2WTRA and T2WSAG) for prostate delineation, covering the entire prostate and seminal vesicles (slice thickness $3 \mathrm{~mm}$, in-plane resolution $0.6 \times 0.7 \mathrm{~mm}^{2}$, see Additional file 1, Table A1), and a balanced turbo field echo acquisition with fat suppression, i.e. spectral attenuated inversion recovery (referred to as FMimage) to visualize the FMs (slice thickness $1 \mathrm{~mm}$, in-plane resolution $1.0 \times 1.0$ $\mathrm{mm}^{2}$ ).

The FMimage was used to facilitate a FM-based registration of the T2WTRA to the CT in our clinical workflow, because FM were hardly visible on the T2WTRA (Fig. 2). First, the T2WTRA was automatically rigidly registered to the FMimage using mutual information as metric and a rectangular region of interest (ROI) containing the prostate but no bony structures (labelled FMimage-T2WTRA). Then, the FMimage was manually registered to the CT, by aligning the FMs (labelled FMimage-CT). Lastly, the CT and the TW2TRA scans were registered by performing the former two registrations in succession (labelled CT-T2WTRA, see Fig. 2). All registrations were performed in Velocity, R4, Varian Medical Systems.

A radiation oncologist contoured the CTV (consisting of the prostate and base of the seminal vesicles) on the T2WTRA, which was fused with the CT using the CTT2WTRA registration, according to the EAU-ESTRO guidelines for prostate cancer [28]. For some patients, elective lymph node regions were also included and contoured by the radiation oncologist on CT images. Specialized radiation therapists (RTTs) contoured the OAR (rectum, anal canal, bladder and both hips, as well as the sigmoid, small bowel and bowel bag in proximity of the PTV) on CT images according to department's protocol.

Different dose prescriptions were used per patient, depending on the classification of the prostate cancer. The treatment planning technique consisted of $10 \mathrm{MV}$ dual arc volumetric modulated arc therapy (VMAT) (Raystation, R7, RaySearch Laboratories).
The IGRT was based on daily online registration using CBCT (slice thickness $0.5 \mathrm{~mm}$, in-plane resolution $0.5 \times 0.5 \mathrm{~mm}^{2}$ ). An automatic bone match was performed to evaluate the patient set-up with a ROI containing the small pelvis, followed by an automatic FM match (XVI, R5.0.4, Elekta).

\section{Simulated MR-only workflow}

The MR-only workflow was simulated by replacing the $\mathrm{CT}$ with the $\mathrm{SCT}$ (see Fig. 1, simulated MR-only workflow in red).

To generate the sCT an additional mDIXON fast field echo (FFE) MRI sequence (slice thickness $2.5 \mathrm{~mm}$, inplane resolution $1.7 \times 1.7 \mathrm{~mm}^{2}$, other clinical MRI scan acquisition parameters can be found in Additional file 1, Table A1) was acquired, sliced in the transverse direction (MRCAT, RTgo plugin 3.0, Philips, 2016). From the mDIXON scan the in-phase, water and fat reconstructions were used to generate the sCT. FOV of the image was from L4/L5 to the caudal border of the symphysis, covering the entire body contour in AP and RL directions. MRCAT is an algorithm using the mDIXON and a model based segmentation of the bones to create a sCT that consists of five different materials: air, compact and spongy bone, fat and water-rich tissue [3]. The mDIXON and the T2WTRA were acquired in succession to minimize organ motion between the scans. From the mDIXON acquisition a water-only image (mDIXON-w) was reconstructed, on which the FM were visible as signal voids (see Fig. 3a). The T2WTRA was registered to the mDIXON-w using an automated registration based on gray values with mutual information as metric and a rectangular ROI containing the prostate without including bony structures. If necessary, the automatic registration was manually adjusted based on the signal voids of the FM on the MRI. Because the sCT was generated from the mDIXON, the T2WTRA image and the $\mathrm{SCT}$ registration was identical to the T2WTRA to mDIXON-w registration (labelled sCT-T2WTRA), shown in Fig. 2.

The FMs are not visible on the $\mathrm{sCT}$ scan, since it identifies only five materials. To automatically identify the FMs on the mDIXON-w scan is challenging without any prior input on the positions of the FM, potentially giving rise to wrongly identification of features showing up as a signal void (e.g., calcifications) [29]. Therefore, we developed a semi-automatic method to identify and burn-in the FM on the sCT. In the first manual step, a dedicated MRI sequence (FMimage) was registered to the mDIXON-w scan to help identify FM positions on the mDIXON-w scan. Once identified, the signal void associated with a FM was manually delineated on three slices of the mDIXON-w scan and isotropically expanded by $2 \mathrm{~mm}$. In the second automatic step, the 
six voxels with the lowest intensity within the ROI were determined and were assigned a CT number of 3000 Hounsfield Units (HU). Using an in-house developed $\mathrm{C}++$ software tool, the FMs were burned-in on the sCT (Fig. 3a-e).

To enable image registration of the sCT with CBCT for position verification a copy RT plan was created on the sCT (RayStation). Image registration for IGRT position verification was performed in the same way as for the CT/MR workflow (XVI, Elekta) (Fig. 1).

To compare the accuracy of the CT/MR- and the MR-only workflow three sources of inaccuracy were investigated:

1. the inter-observer variation of the image registration for target delineation (CT-T2WTRA versus SCTT2WTRA),

2. the inter-observer variation of burned-in FM positions using the semi-automatic method (only in MRonly workflow),

3. the accuracy of FM-based image registration for position verification (CT to $\mathrm{CBCT}$ versus $\mathrm{SCT}$ to CBCT).

\section{The inter-observer variation of image registration for target delineation}

Seven experienced observers (five RTTs and two medical physicists) performed image registration for target delineation for both the CT/MR workflow (CT-T2WTRA) and the MR-only workflow (sCT-T2WTRA) for the first twenty patients consecutively included in this study. The resulting registrations, which were labelled CTT2WTRA for the CT/MR and SCT-T2WTRA for the MR-only workflow (Fig. 2), were specified by six parameters: the translation in right-left (RL), cranio-caudal (CC), and anterior-posterior (AP) direction, and the rotation angle about the RL, CC, and AP axis. For each parameter the inter-observer registration error (IOE) was quantified as the variation of registration results. For the individual patients, the inter-quartile range (IQR) was calculated. To be able to pool the data between patients, the mean of the registration values per patient was subtracted. For the cohort of patients, the IOE of the registration parameters was described by the standard deviation (SD).

A paired two-sided Wilcoxon signed-rank test with a significance level of $5 \%$ was used to test the difference in IQR. A non-parametric Levene's test with a significance level of 5\% was used to test the difference in SDs for the registration results of the pooled data, e.g. for all observers and patients combined (data analyzed with IBM SPSS Statistics for Windows, Version 24.0 (NY: IBM Corp)).

\section{The inter-observer variation of burned-in FM positions} using the semi-automatic method

The seven observers (five RTTs and two medical physicists) delineated the signal voids associated with the FM on the mDIXON-w image for the first twenty patients consecutively included in this study It was determined whether all observers were able to agree in correctly identifying the same FMs. Then the center of mass (COM) of the $1,2,3$ up to 15 voxels with the lowest intensity within each delineation was calculated.

To quantify the inter-observer variation for each marker the SD of the RL, CC and AP component of the COM position was calculated. The SD of the FM positions for 1-15 burned-in voxels were compared.

\section{The accuracy of FM-based image registration for position verification}

All available CBCT scans were included of patients in this study for whom the generation of the $\mathrm{SCT}$ was successful. As described earlier, signal voids caused by the FM were delineated and six voxels within a $2 \mathrm{~mm}$ expanded region around the delineation burned-in on the sCT. These delineations were created by an experienced RTT and checked by a medical physicist. The sCT with burnedin FM was retrospectively registered with the CBCT by an experienced RTT in the same way as for the CT/MR workflow. The CT/MR workflow was considered the gold standard for image registration for position verification.

For a proper comparison of $\mathrm{SCT}-\mathrm{CBCT}$ and $\mathrm{CT}-\mathrm{CBCT}$ registrations, the different patient position and anatomy on $\mathrm{SCT}$ and CT needed to be taken into account. This was achieved by correcting the $\mathrm{SCT}-\mathrm{CBCT}$ registrations using a FM-based registration between $\mathrm{CT}$ and $\mathrm{sCT}$ using an in-house developed software tool (Additional file 1). Remaining discrepancies between FM positions on $\mathrm{SCT}$ and CT after this rigid registration were caused by prostate deformation and inaccuracies in determining center of mass (COM) positions of the FMs on the different modalities, and were quantified by calculating the root mean square (RMS) of the components of the difference vector of the COM positions of all markers of all patients.

After this correction was applied for each patient, the average and $\mathrm{SD}$ of the translation in $\mathrm{RL}, \mathrm{CC}$, and $\mathrm{AP}$ direction, and the rotation angle about the $\mathrm{RL}, \mathrm{CC}$, and $\mathrm{AP}$ axis of the available $\mathrm{CBCT}$ registrations were calculated for both CT/MR and MR-only workflow. These were used to determine population mean, systematic, and random error per workflow. The distributions for the $\mathrm{CT} / \mathrm{MR}$ and MR-only workflow were compared by using a non-parametric Wilcoxon signed-rank test with a significance level of $5 \%$, to evaluate whether the difference 
Table 1 Registration results and the significance of the differences in inter-observer variation between the MR-only workflow (sCTT2WTRA) and CT/MR workflow (CT-T2WTRA) on patient and group level

\begin{tabular}{|c|c|c|c|c|c|c|}
\hline & \multicolumn{3}{|c|}{ Per patient comparison } & \multicolumn{3}{|c|}{ Per group comparison } \\
\hline & CT-T2WTRA & sCT-T2WTRA & $p$-value & CT-T2WTRA & sCT-T2WTRA & p-value \\
\hline & $\begin{array}{l}\text { Mean IQR } \\
(\mathrm{mm})\end{array}$ & $\begin{array}{l}\text { Mean IQR } \\
(\mathrm{mm})\end{array}$ & & $\begin{array}{l}\text { SD } \\
(\mathrm{mm})\end{array}$ & $\begin{array}{l}\text { SD } \\
(\mathrm{mm})\end{array}$ & \\
\hline \multicolumn{7}{|c|}{ Translation } \\
\hline $\mathrm{RL}$ & 0.96 & 0.30 & 0.001 & 0.87 & 0.25 & $<0.001$ \\
\hline CC & 1.48 & 0.37 & $<0.001$ & 1.26 & 0.30 & $<0.001$ \\
\hline AP & 1.58 & 0.48 & $<0.001$ & 1.32 & 0.40 & $<0.001$ \\
\hline \multicolumn{7}{|l|}{ Rotation } \\
\hline RL-axis & 2.52 & 0.66 & $<0.001$ & 1.97 & 0.59 & $<0.001$ \\
\hline CC-axis & 1.19 & 0.50 & 0.001 & 1.17 & 0.42 & $<0.001$ \\
\hline AP-axis & 1.19 & 0.43 & 0.002 & 1.18 & 0.43 & $<0.001$ \\
\hline
\end{tabular}

\section{CT/MR workflow}

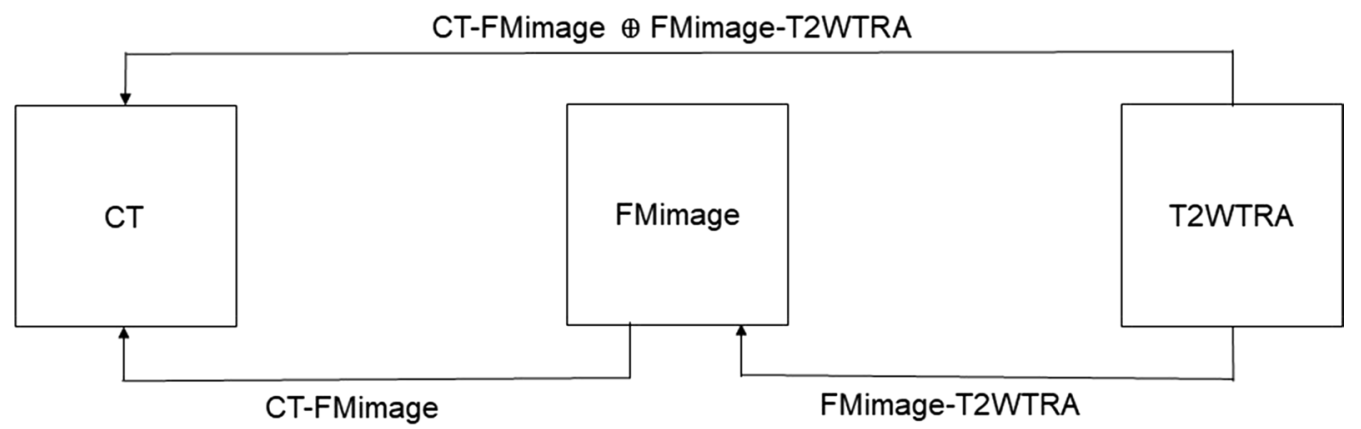

MR-only workflow

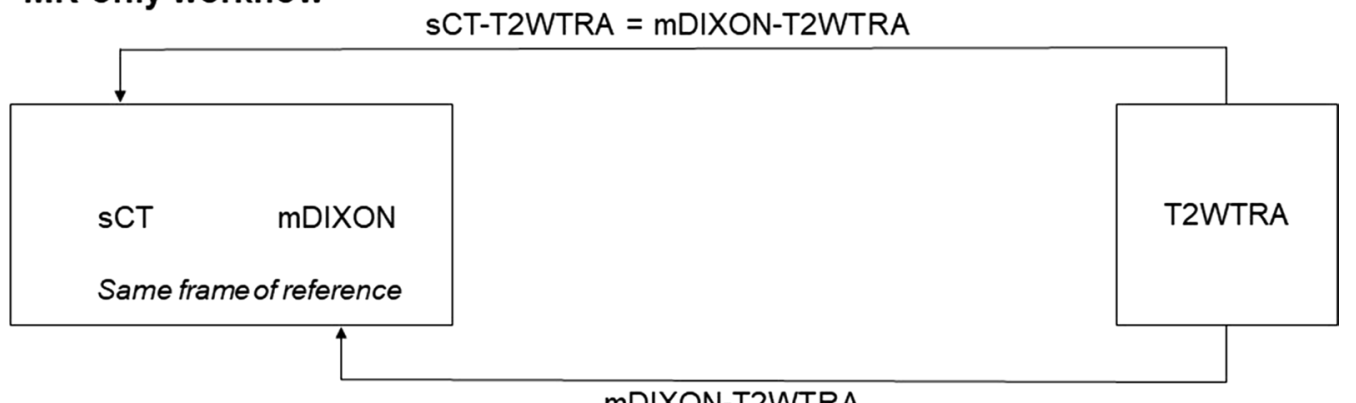

mDIXON-T2WTRA

Fig. 2 Pre-treatment imaging registration in Velocity for the CT/MR and the MR-only workflow. For proper tumor delineation, CT-T2WTRA should be coregistrated based on the FM positions. However, FMs are not visible on the T2W image. A FMimage was acquired on which the FMs are visible. CT-FMimage registration was based on FMs, and FMimage-T2WTRA registration was based on prostate anatomy. The final CT-T2WTRA registration results from combining the two registrations. On the mDIXON image FM are visible. The SCT was generated from the mDIXON acquisition and share the same frame of reference. The final SCT-T2WTRA registration is identical to the mDIXON-T2WTRA registration because the sCT and mDIXON are intrinsically registered 


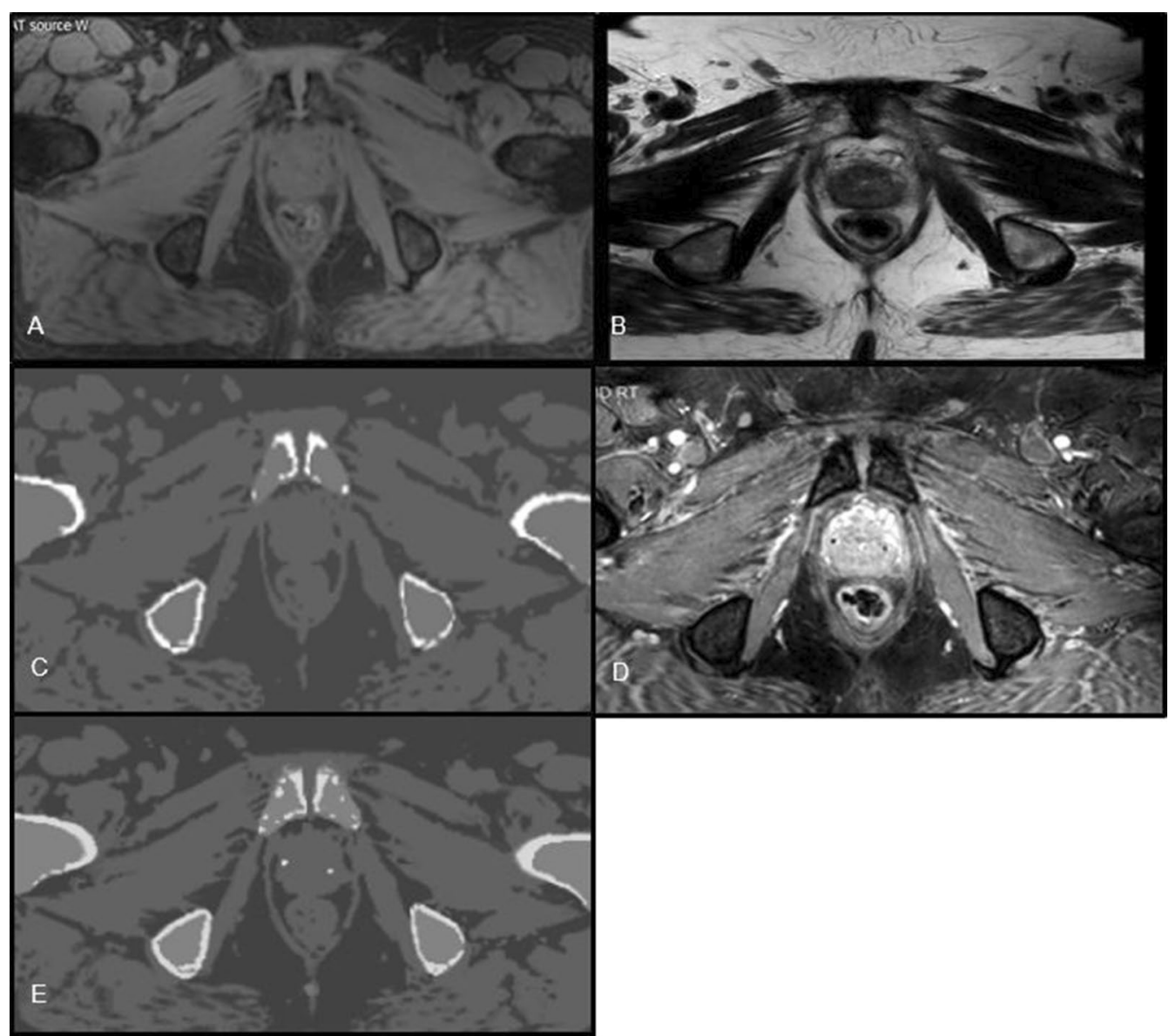

Fig. 3 Overview of a semi-automatic method for burning- in the FM on the $S C T$. Images A-E in a transversal view of a: mDIXON-w with FMs visible as signal voids, $\mathbf{b}$ : T2WTRA image used for registration with mDIXON-w. c: sCT reconstructed from an mDIXON-w acquisition used for delineating the marker position, d: a BFTE SPAIR sequence used to help finding the FM positions (FMimage) and E: a sCT with burned-in FM used for IGRT

in distributions between both workflows was statistically significant (SPSS).

Additionally, for each CBCT the difference between the sCT-CBCT and CT-CBCT registration was determined. For each patient the average and SD of the difference in translation in RL, CC and AP direction, and rotation angle about the RL, CC and AP were calculated for the available $\mathrm{CBCT}$ registrations of this patient. These were used to determine population mean, systematic and random errors. A non-parametric Wilcoxon signed-rank test with a significance level of $5 \%$ was used to test whether population mean was different from zero (SPSS).

\section{Results}

Thirty-one patients were included in the simulated MRonly workflow. The generation of the sCT failed in four patients and a fifth patient was imaged in the wrong position and therefore analysis was not possible. Twenty patients were included in the inter-observer sub-studies and twenty-six patients were included in the accuracy of FM-based image registration for position verification sub-study.

\section{The inter-observer variation of image registration for target delineation}

For twenty patients, seven observers performed the CTT2W and SCT-T2W registration for target delineation. The IOE for CT-T2WTRA and SCT-T2WTRA of the mean IQR per patient and the SD per group can be found in Table 1. In general, the IOEs of SCT-T2WTRA (MRonly workflow) were about three times smaller for both translations and rotations in $\mathrm{RL}, \mathrm{CC}$ and $\mathrm{AP}$ direction compared to CT-T2WTRA (CT/MR workflow).

As an example, the variation of RL translation and rotation is shown in Fig. 4a, b, where the mean RL translation and rotation per patient was set to zero. Each box shows the results of the seven observers, the height of the box is indicative for the inter-observer variation. 


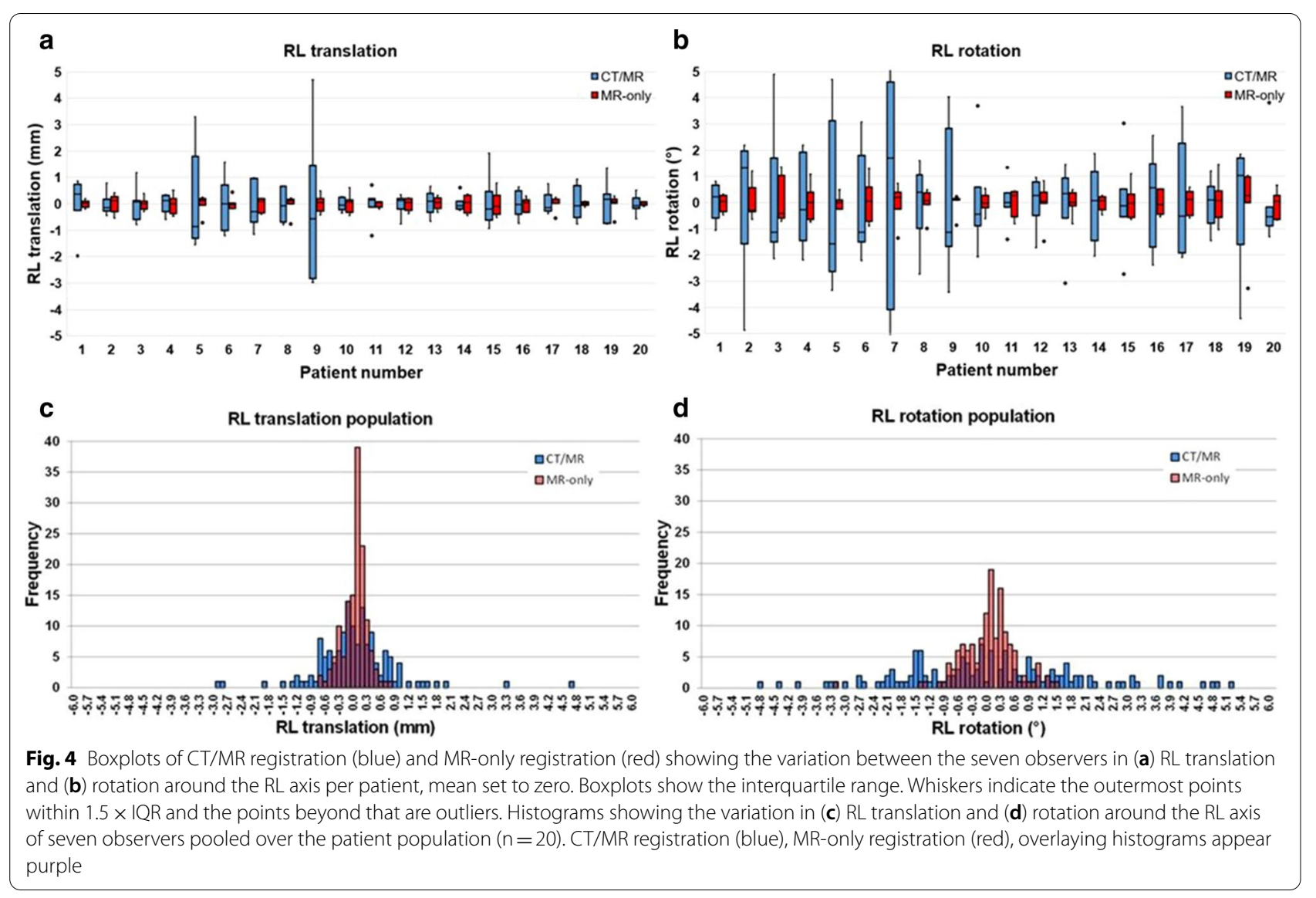

The inter-observer variation for the MR-only workflow (sCT-T2WTRA, red) was significantly smaller $(\mathrm{p}<0.001)$ than for the CT/MR workflow (CT-T2WTRA, blue). The mean IQR for RL translation of the CT/MR workflow was $0.96 \mathrm{~mm}$ and for the MR-only workflow $0.30 \mathrm{~mm}$ (see Table 1).

The variation in RL translation of seven observers pooled over the patient population $(n=20)$ is shown in Fig. 4c, d. The inter-observer variation in an MR-only registration (red) was about three times smaller $(\mathrm{p}<0.001)$ compared to the CT/MR based registration (blue). The overlaying histograms appear purple.

The SD per group for RL translation of the CT/MR workflow was $0.87 \mathrm{~mm}$ and for the MR-only workflow $0.25 \mathrm{~mm}$ (Table 1). The other registration parameters showed similar behaviour, and are shown in Figure D1D2 in the Additonal file 1.

\section{The inter-observer variation of burned-in FM positions using the semi-automatic method}

At the time of the examination 2 out of 20 patients had 3 FMs instead of 4 FMs, amounting to a total of 78 FMs. All observers were able to identify all FMs correctly for eighteen patients. For the two patients with three FMs all observes agree in correctly identifying three FMs. Yet for one of the two patients six observers identified a nonexistent fourth marker, and for the other patient three observers identified a non-existent fourth marker. The level of the observers agreement in correctly identifying the same FM for the patients in our study is as follows: five observers agree that they correctly identifying the same FM for $95 \%$ of the patients and two observers agree that they correctly identify the same FM for $90 \%$ of the patients.

A cumulative histogram of the SDs of the COM positions of the markers defined by seven observers are shown in Fig. 5 for one, six and twelve voxels burned-in voxels.

For $96 \%$ of the markers, the SD of the RL and AP component of the COM position was less than $0.5 \mathrm{~mm}$, regardless of the number of burned-in voxels. For one marker the SD of the CC component (slice direction) of the COM position was $4.5 \mathrm{~mm}$ if one voxel was burned-in, which was the largest SD and resulted from a misidentified marker. For $94 \%, 90 \%$ and $87 \%$ (Fig. 5) of the markers the SD of the CC component of the COM position was less than $0.5 \mathrm{~mm}$ for one, six, and twelve burned-in voxels, respectively, which is in the realm of 

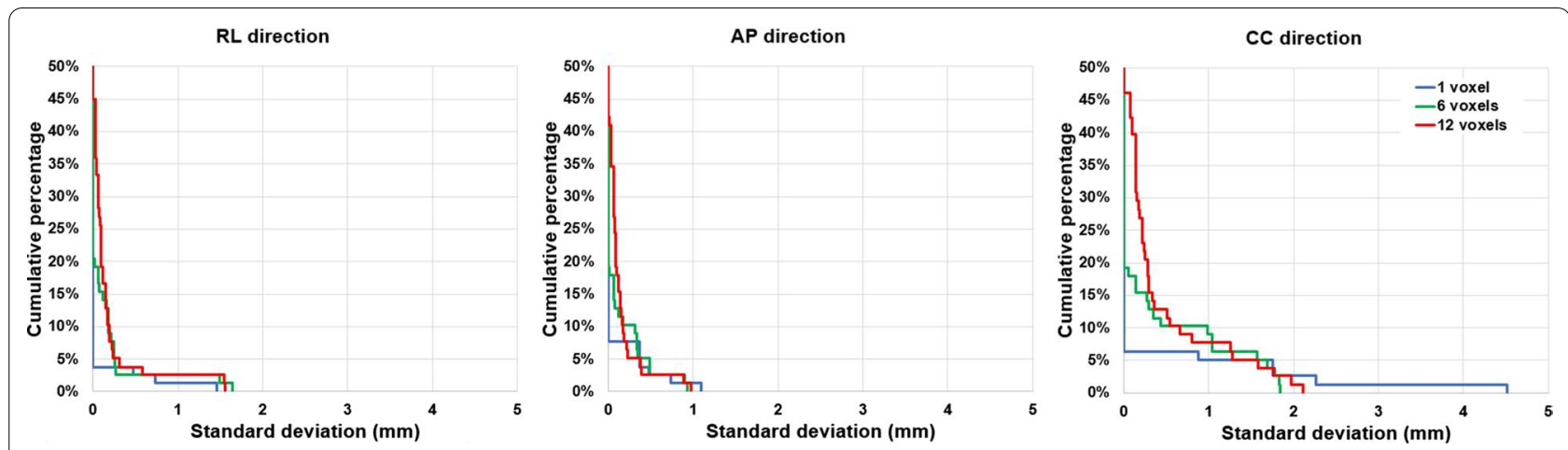

Fig. 5 Cumulative histograms of the SD of the RL-, CC- and AP-component of the COM position of FMs consisting the one (blue), six (green) and twelve (red) voxels with the lowest signals. The vertical axis shows the percentage of FMs with a SD more than the value given on the horizontal axis. Example: for the SD of AP for 3\% of the FMs the SD of the AP-component of the FM position was larger than $0.5 \mathrm{~mm}$

the slice resolution of $2.5 \mathrm{~mm}$. Using six voxels to burn in the FM resulted in a maximum SD for the CC component of $2.2 \mathrm{~mm}$.

\section{The accuracy of FM-based image registration for position verification}

We analysed 618 CBCTs of 26 patients. The CBCTs were registered with the $\mathrm{sCT}$ using burned-in FMs (average of 24 CBCTs per patient, range 12-35). Three patients were treated on prostate and elective lymph node regions, followed by radiotherapy on the prostate alone. Registration of the prostate with elective lymph node regions was based on bony pelvic anatomy and therefore not included in this study.

An overview of the results of the (burned-in) FM position verification between $\mathrm{SCT}-\mathrm{CBCT}$ and CT-CBCT is shown in Table 2. The population mean for the CT/MR workflow and the MR-only workflow is similar in all directions, with a maximum of $0.1 \mathrm{~mm}$ difference. The systematic error in RL translation of the CT/MR workflow and MR-only workflow was $2.8 \mathrm{~mm}$. The random error in RL translation was $3.3 \mathrm{~mm}$ for both workflows.

The results of the simulated MR-only workflow (sCT$\mathrm{CBCT}$ ) and $\mathrm{CT} / \mathrm{MR}$ workflow (CT-CBCT) concerning population mean, systematic error and random error for translations in RL-, CC- and AP direction did not differ significantly. The random error for rotation about RL-axis $(\mathrm{p}<0.001), C C$-axis $(\mathrm{p}<0.001)$ and AP-axis $(\mathrm{p}<0.001)$ differed significantly. Boxplots of the results of image registration for position verification of the translation and rotation in RL direction of 26 patients for the MR-only workflow and CT/MR workflow are shown in Fig. 6a, b. The results of image registration for position verification of the other registration parameters (translation in CC and AP direction and of the rotation in CC and AP direction) are shown in Figure D3 in the Additonal file 1.

An overview of the results of the difference between the registration of the simulated MR-only workflow and CT/MR workflow with CBCT ((sCT-CBCT) - (CT$\mathrm{CBCT}$ )) in translations and rotations in $\mathrm{RL}, \mathrm{CC}$ and $\mathrm{AP}$ direction can be found in Table 3. The population mean did not differ significantly from zero for both translations and rotations. The systematic error and random error in $\mathrm{RL}$ direction of the difference between the registration of sCT and CT with each CBCT was $0.2 \mathrm{~mm}$ and $0.3 \mathrm{~mm}$, respectively (Table 3).

The residual error of the FM registration between $\mathrm{SCT}$ and $\mathrm{CT}$ in translations in RL, CC and AP direction was below $1 \mathrm{~mm}(0.5 \mathrm{~mm}$ in RL, $0.8 \mathrm{~mm}$ in $\mathrm{CC}$ and $0.2 \mathrm{~mm}$ in AP direction), of which the largest component is in the slice (CC) direction. The residual error of the FM registration is within the same range of differences found in

Table 2 Position verification results for the MR-only workflow and the CT/MR workflow, based on burned-in FM. Values indicated by an asterisk are significantly different from zero ( $p$-value $<0.05)$

\begin{tabular}{|c|c|c|c|c|c|c|c|c|c|c|c|c|}
\hline & \multicolumn{2}{|c|}{$\mathrm{RL}(\mathrm{mm})$} & \multicolumn{2}{|c|}{$\mathrm{CC}(\mathrm{mm})$} & \multicolumn{2}{|c|}{$\mathrm{AP}(\mathrm{mm})$} & \multicolumn{2}{|c|}{ RL-axis $\left(^{\circ}\right)$} & \multicolumn{2}{|c|}{$\mathrm{CC}$-axis $\left(^{\circ}\right)$} & \multicolumn{2}{|c|}{ AP-axis $\left({ }^{\circ}\right)$} \\
\hline & $\mathrm{sCT}$ & CT & sCT & CT & $\mathrm{sCT}$ & CT & $\mathrm{sCT}$ & CT & sCT & CT & sCT & CT \\
\hline Population mean & -0.2 & -0.3 & -0.7 & -0.7 & -0.3 & -0.3 & -0.5 & -0.6 & 0.8 & 0.6 & -0.6 & -0.5 \\
\hline Systematic error $(\Sigma)$ & 2.8 & 2.8 & 3.8 & 3.7 & 2.9 & 2.8 & 4.6 & 4.8 & 2.7 & 2.6 & 2.4 & 1.8 \\
\hline Random error ( $\sigma)$ & 3.3 & 3.3 & 2.7 & 2.7 & 2.9 & 2.8 & $4.4^{*}$ & $4.0^{*}$ & $2.2^{*}$ & $1.9^{*}$ & $1.4^{*}$ & $1.2^{*}$ \\
\hline
\end{tabular}



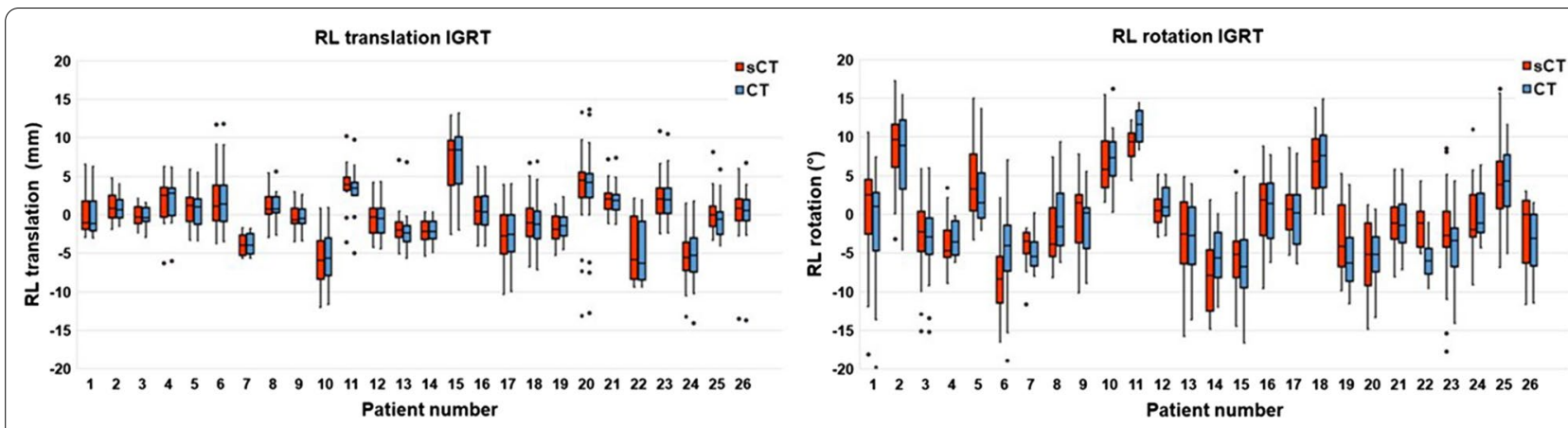

Fig. 6 Boxplots of the IGRT registrations of SCT-CBCT (red) and CT-CBCT (blue). Distributions of (a) the translation in RL direction and (b) rotation over the RL axis over the patient population $(n=26)$ are shown. Boxplots shows the interquartile range. Whiskers indicate the outermost points within $1.5 \times \mathrm{IQR}$ and the points beyond that are outliers

Table 3 Difference between the registration of sCT and CT with CBCT

\begin{tabular}{|c|c|c|c|c|c|c|}
\hline & $\mathrm{RL}(\mathrm{mm})$ & $\mathrm{CC}(\mathrm{mm})$ & $\mathrm{AP}(\mathrm{mm})$ & RL-axis $\left(^{\circ}\right)$ & CC-axis $\left(^{\circ}\right)$ & AP-axis $\left({ }^{\circ}\right)$ \\
\hline Population mean & 0.1 & 0.0 & 0.0 & 0.2 & 0.1 & -0.2 \\
\hline Systematic error $(\Sigma)$ & 0.2 & 0.4 & 0.4 & 1.7 & 1.1 & 1.1 \\
\hline Random error ( $\sigma)$ & 0.3 & 0.5 & 0.6 & 2.5 & 2.1 & 1.2 \\
\hline
\end{tabular}

the systematic and random errors between the $\mathrm{SCT}$ CBCT and CT-CBCT registration.

\section{Discussion}

In this study, three sources of inaccuracies of a simulated MR-only workflow using a sCT with semi-automatically burned-in FMs were compared to the conventional CT/ MR workflow. From these results we can conclude that an MR-only workflow with IGRT based on burned-in FMs can be safely introduced.

Firstly, the inter-observer variation of the image registration for target delineation was found to be a factor of three smaller for the simulated MR-only workflow, as compared to the CT/MR workflow. Nyholm et al. [13] showed that the systematic uncertainties introduced by CT/MR-registration were reduced with the MR-only workflow from about 3 to $4 \mathrm{~mm}$ to about 2 to $3 \mathrm{~mm}$, primarily by the omission of the $\mathrm{CT} / \mathrm{MR}$ registration in the RTP. In our study, the IOE was reduced from about 0.9 to $1.3 \mathrm{~mm}$ to about 0.3 to $0.4 \mathrm{~mm}$. The random uncertainties were approximately the same for the MR-only workflow as for the CT/MR workflow [13]. The reduction of IOE of image registration for target delineation in our study are likely due to the shorter time period between the various MRI acquisitions (minutes) compared to the up to $2 \mathrm{~h}$ time period between the CT and MRI examinations. This shorter time period allows for less organ motion, which makes registration more accurate. The IOE of the MR-only workflow is based on a T2WTRA to
mDIXON-w registration (the same frame of reference as the $\mathrm{sCT}$ ) and the soft tissue registration was assessed for the prostate. Whereas for the IOE of the CT/MR workflow, a FM match was performed manually in Velocity introducing inter-observer variation, which is probably caused because FMs display differently, on CT images as large as an artefact and on MRI images as a small signal void.

However, further reduction of errors caused by image registrations is still possible. In our study, we used several MRI sequences in the MR-only workflow. An mDIXON scan, including in-phase, water and fat reconstructions, was needed to generate a $\mathrm{sCT}$. The water image of this mDIXON scan clearly showed the FMs and could therefore also be used for FM identification. However, a T2W scan was best for depicting target area and therefore enabled more accurate delineation of the target area. Therefore an automated soft tissue intra-MRI registration of T2WTRA to mDIXON-w was needed, introducing a small registration error. Ideally only one sequence is used for $\mathrm{SCT}$ generation, target and OAR delineation and identifying FMs and burning in FMs for IGRT.

The PTV margin is calculated taking into account various uncertainties in the radiotherapy workflow such as delineation errors, registration uncertainties, deformation of the anatomy and intrafraction motion, to name a few. Based on this presented work we know that the inter-observer error in the CT-MR registration is reduced by a factor of three. However, how large this 
effect is compared to other uncertainties contributing to the PTV margin is outside the scope of this article.

Secondly, the results of the inter-observer variation of burning-in FM positions showed that all seven observers agree that they were correctly identifying the same four FMs for 18 out of 20 patients. Five observers have a $95 \%$ level of observers agreement in correctly identifying the same FM for the patients in our study, two observers have a $90 \%$ level of observers agreement. The IOE in CC direction of the COM position of the markers defined was within the slice resolution of the $\mathrm{CT}$ $(2.5 \mathrm{~mm})$, the IOE in AP and RL direction were below $1.0 \mathrm{~mm}$ and $1.5 \mathrm{~mm}$, respectively.

A (semi)-automatic method for FM identification on MRI was used by several research groups [2, 14, 20,24]. The results of these methods were promising, resulting in acceptable accuracy and relatively high FMs identification rates (81\%-96\%). However, manual observation by radiotherapy staff remained necessary to correct for missed FM [2, 7, 20, 24,31]. For two patients in this study, with three instead of four FMs, not all observers agree in correctly identifying the correct number of FMs. The detection performance of automatic FM identification is typically less than $100 \%$. Visual inspection of the outcome of the automatic FM identification by another observer is therefore recommended, to prevent misidentification of FMs [1, 20, 24]. In more than $40 \%$ of prostate cancer patients intraprostatic calcifications are present, having similar appearance as a FM on MRI [29]. The combination of different MRI sequences with dedicated sequences intended for FM identification (e.g. a multi-echo gradient echo, MEGRE, or a balanced Turbo-Field Echo, bTFE), can be used in the MR-only workflow to distinguish FMs from calcifications, but also blood clots or air pockets [7, 20, 24].

Automatic quality control [2] and MR-based automatic FM identification [20] systems used to identify FMs have successfully been developed in the past.

In a recently published study by Tenhunen et al. [14] in which 200 prostate cancer patients were treated with an MR-only workflow, $92 \%$ of the treatments were successful, in the remaining $8 \%$ there was no certain identification of the FMs on MRI [14]. Comparable accurate identification of FMs in a MR only approach $(81 \%)$ have been described by Dinis Fernandes et al. [7].

The inter-observer variation of the COM positions of the FMs was in the order of the scan resolution $\left(1 \times 1 \times 2.5 \mathrm{~mm}^{3}\right)$, and was largest in the slice direction. This is in line with the results found by Persson et al. [34], they found the largest range in deviations in marker distances to the respective centroid in $\mathrm{SCT}$ and $\mathrm{CT}$ in the slice direction [34].
In our study, we chose to burn-in six voxels on the sCT. On the CT typical 10-15 voxels (scan resolution $1 \times 1 \times 2.5 \mathrm{~mm}^{3}$ ) consist of the highest $\mathrm{HU}$ values, corresponding with 3-5 sCT voxels (scan resolution $1.7 \times 1.7 \times 2.5 \mathrm{~mm}^{3}$ ). Using six voxels seems to be a feasible choice and also results in a small IOE of the COM position. Burning in less voxels increased the maximum $\mathrm{SD}$ of the CC-component of the COM position, which becomes smaller when including more voxels (Fig. 5). Burning in more voxels gives rise to a larger reconstructed FM which does not reflect the physical size of the FM: a cylinder with $1 \mathrm{~mm}$ diameter and $5 \mathrm{~mm}$ length.

FMs implanted very near to each other could be more difficult to distinguish and cause a difference of COM positions of the FMs between observers. In this study the $\mathrm{SD}$ of the $\mathrm{CC}$ component of the $\mathrm{COM}$ position of one marker was $4.5 \mathrm{~mm}$ if one voxel was burned-in, which was the largest SD and resulted from a misidentified marker. Future development of automatic FMs identification may result in reduced workload within the radiotherapy department. Commercial software support for automatic burned-in FM may be necessary for widespread adaptation of an MR-only workflow.

Other acquisition parameters could be deployed for scanning an FMimage, which we have not explored in this study.

Thirdly, using burned-in FM for the MR-only workflow for registration with $\mathrm{CBCT}$ for position verification did not differ significantly compared to the CT/MR workflow, except for the random error of the RL, CC and AP rotation angles, which was slightly larger for the MR-only workflow. Several research groups have used this method as well [25]. Tyagi et al. [25] showed results of FM-based manual registration of $\mathrm{CBCT}$ to $\mathrm{SCT}$ or $\mathrm{CT}$ for five hypofractionated prostate cases with five fractions each (a total of 25 CBCTs) based on translation only. The mean translation differences were less than $1 \mathrm{~mm}$ for RL and $\mathrm{AP}$ and less than $0.5 \mathrm{~mm}$ for the CC direction. The standard deviations (SD) were $0.79 \mathrm{~mm}, 0.85 \mathrm{~mm}$ and $0.89 \mathrm{~mm}$ along RL, CC and AP directions, respectively. The mean differences of our accuracy of image registration of $\mathrm{sCT}$ with $\mathrm{CBCT}$ using burned-in FMs were $0.1 \mathrm{~mm}, 0.0 \mathrm{~mm}$ and $0.0 \mathrm{~mm}$ in RL, CC and AP directions. The SD were $0.2 \mathrm{~mm}, 0.4 \mathrm{~mm}$ and $0.4 \mathrm{~mm}$ in RL, CC and AP directions. These results are smaller comparing the results of Tyagi et al., probably due to a more consistent FM-based automatic chamfer matching algorithm that we used, compared to the FM-based manual registration performed by Tyagi.

Maspero et al. [20] used root mean square of the residual error of the FM registration between $\mathrm{sCT}$ and $\mathrm{CT}$ to assess the quality of the registrations. In cases were the residual error was larger than $2.5 \mathrm{~mm}$, the 
quality of registration was considered poor. For one patient only a poor registration quality was found, the other quality of registrations, only taking into account translation, were below $2.5 \mathrm{~mm}$ and comparable for the $\mathrm{SCT}$ and $\mathrm{CT}$ method [20]. In our study the population RMS was below $1 \mathrm{~mm}$ for RL, CC and AP direction. Assessing these values in quality of registration according to Maspero's method, the quality can be considered good. The differences between the $\mathrm{SCT}-\mathrm{CBCT}$ and CT-CBCT registrations were in the order of the residual error between the $\mathrm{CT}$ and $\mathrm{sCT}$. This gives confidence that the MR-only workflow is as accurate as the CT/MR workflow.

Ideally patients on the MRI are imaged with a comparable bladder filling, as used in the CT workflow and during treatment. Because it was not yet clear whether the FOV of the MR sequences was large enough, study patients were imaged with an empty bladder on the MRI. As a result, patients included in this study have different bladder filling on the sCT (empty bladder) compared to the CBCT (filled bladder).

We performed this study at a $3 \mathrm{~T}$ MRI since it was the type of scanner available at our department. $3 \mathrm{~T}$ MRI outperforms 1.5 T MRI for DWI and subjective image quality [30]. The SNR is typically higher though the images are more prone to geometrical uncertainties. However, the vendor-provided 3D mDixon acquisition has a geometrical accuracy at the level of the body contour of less than $1 \mathrm{~mm}$ [25]. For the clinical protocols, SAR (specific absorption rate) limitations were no issue.

A practical hurdle to the widespread adoption of an MR-only workflow is the failure to generate a sCT [1]. In our study the generation of the $\mathrm{SCT}$ failed for four out of thirty-one patients. Failures in the reconstruction were caused by scanning the patient in an abnormal position (skewed, femoral heads not at the same height, arms along the body) and in another patient artefacts occurred. If a sCT cannot be generated, a $\mathrm{CT} / \mathrm{MR}$ workflow should remain available to allow the radiotherapy to proceed.

This study was conducted based on a sCT with 5 different materials each of a different HU. Kemppainen et al. [30] has shown that segmentation of anatomy into 5 grey value levels is dosimetrically accurate enough to produce clinically acceptable accuracy in dose calculation for prostate cancer patients treated with external beam radiotherapy. This dosimetric study will not be discussed further in this study. However, it is necessary to further investigate on burning-in FMs into the accuracy of the prostate radiotherapy chain based on a continuous grayscale $\mathrm{sCT}$.

\section{Conclusion}

MR-only workflow for prostate cancer reduces the inter-observer variation of the image registration for target delineation by a factor of three, compared to the current standard CT/MR workflow. The inter-observer variation of the burned-in FM positions by our semiautomatic method was in the order of the scan resolution. CBCT registration in our MR-only workflow using burned-in FMs is feasible and has comparable accuracy as our current CT/MR workflow. Therefore, an MR-only workflow using sCT with burned-in FMs is an improvement compared to the current workflow for prostate cancer patients.

\section{Abbreviations}

B0 map: Measurement of the local magnetic field per voxel, and used to correct for magnetic field inhomogeneities; CBCT: Cone Beam CT; COM: Center Of Mass; COR: Coronal; CT: Computed Tomography; CTV: Clinical Target Volume; DICOM: Digital Imaging and COmmunications in Medicine; EBRT: External Beam RadioTherapy; FMimage: An MR sequence that is used to identify the FMs; FMs: Fiducial Markers; GTV: Gross Tumor Volume; Gy: Gray; HU: Hounsfield Units; IGRT: Image-Guided Radiation Therapy; IOE: Inter-Observer Error; IQR: Inter Quartile Range; KV: KiloVolt; mDIXON FFE: MRI sequence used for fat suppression and/or fat quantification; MR(I): Magnetic Resonance (Imaging); MRCAT: Magnetic Resonance for Calculating ATtenuation; MV: MegaVolt; OAR: Organ At Risk; PTV: Planning Target Volume; ROI: Region Of Interest; RTP: Radiation Treatment Planning; RTT: Radiation TherapisT; SAG: Sagittal; SAR: Specific absorption rate; sCT: Synthetic Computed Tomography; SD: Standard Deviation; SPAIR: Balanced turbo-field-echo with SPectral Attenuated Inversion Recovery; FMimage; signal void: Absence of signal on MRI due to metal artifacts (e.g. gold fiducial markers); sROl: Shaped Region Of Interest; THRIVE: T1-weighted High Resolution Isotropic Volume Examination; TRA: Transversal; TSE: Turbo Spin Echo; T1W: T1-Weighted, spin-lattice relaxation time; T2W: T2-Weighted, spin-spin relaxation time; VMAT:Volumetric Modulated Arc Therapy; XVI: X-ray Volume Imaging, R5.0.4, Elekta.

\section{Supplementary information}

The online version contains supplementary material available at https://doi. org/10.1186/s13014-021-01768-8.

Additional file 1. This article contains additional information which support and expand upon items referred to in the main manuscript. These items are included in the file Supplemental_Material.pdf and consist of Supplement A: the MRI-scan acquisition parameters; Supplement B and C: description of the in-house developed software tool to correct for the difference between patient position and anatomy; Supplement D: additional boxplots and histograms.

\section{Acknowledgements}

The authors like to thank Astrid van der Horst for advice on scientific writing style and graphic support. And Sylvia Kleuskens for providing feedback on MRI-related parts of the article.

\section{Authors' contributions}

All authors made substantial contributions to the design of the MR-only workflow clinical protocol and the design of the methods used in this study. $\mathrm{M}$. Boon performed the pre-treatment registrations and JV burned-in the FMs and created simulated treatment plans. KG prepared and performed the registrations of all CBCTs KG, JV, analysed the data and KG drafted the manuscript. All authors have contributed significantly to the manuscript, have read the manuscript and have given final approval of the version to be published, and 
take public responsibility for appropriate portions of the content. All authors read and approved the final manuscript.

\section{Funding}

Not applicable.

\section{Availability of data and materials}

The datasets generated and/or analysed during the current study are not publicly available since the participants did not consent in sharing the data with third parties. The outcome of the study and part of the anonymous data may be shared with Philips Healthcare for further optimization of the sCT.

\section{Ethics approval and consent to participate}

The institutional ethics committee granted an exemption from requiring ethics approval in this study (NL65414.018.18; Academic Medical Center, University of Amsterdam, Amsterdam, NL).

\section{Consent for publication}

Figures depicting individual person's data are unidentifiable and there are no details on individuals reported within the manuscript. Therefore, separate consent for publication is not deemed necessary for the figures shown in this manuscript.

\section{Competing interests}

The authors declare that they have no competing interests. Dr. van Kesteren has research agreements with Varian and Philips Healthcare. Both industries were not a member of the research team and did not influence this study in any way.

Received: 7 December 2020 Accepted: 11 February 2021

Published online: 19 February 2021

\section{References}

1. Tyagi N, Fontenla S, Zelefsky M, Chong-Ton M, Ostergren K, Shah N, et al. Clinical workflow for MR-only simulation and planning in prostate. Radiat Oncol. 2017;12(1):1-12.

2. Ghose S, Mitra J, Rivest-Hénault D, Fazlollahi A, Stanwell P, Pichler P, et al. MRI-alone radiation therapy planning for prostate cancer: Automatic fiducial marker detection. Med Phys. 2016;43(5):2218-28.

3. Köhler M, Vaara T, Grootel M Van, Hoogeveen R, Kemppainen R, Renisch S. White paper: MR-only simulation for radiotherapy planning. Philips [Internet]. 2015;1-16. Available from: http://incenter.medical.philips.com/doclib/ getDoc.aspx?func=\|\&objld=11228039\&objAction=Open

4. Davies. Clinical Applications for Diffusion MRI in Radiotherapy Christina. Semin Radiat Oncol. 2014;24(3):218-26.

5. Rasch C, Barillot I, Remeijer P, Touw A, Van Herk M, Lebesque JV. Definition of the prostate in CT and MRI: A multi-observer study. Int J Radiat Oncol Biol Phys. 1999;43(1):57-66.

6. Khoo VS, Padhani AR, Tanner SF, Finnigan DJ, Leach MO, Dearnaley DP. Comparison of MRI with $\mathrm{CT}$ for the radiotherapy planning of prostate cancer: A feasibility study. Br J Radiol. 1999;72(JUN.):590-7.

7. Dinis Fernandes C, Dinh C V., Steggerda MJ, ter Beek LC, Smolic M, van Buuren LD, et al. Prostate fiducial marker detection with the use of multiparametric magnetic resonance imaging. Phys Imaging Radiat Oncol [Internet]. 2017;1(July 2018):14-20. Available from: http://dx.doi.org/https:// doi.org/10.1016/j.phro.2017.02.001

8. Huisman HJ, Fütterer JJ, Van Lin ENJT, Welmers A, Scheenen TWJ, Van Dalen $J A$, et al. Prostate cancer: Precision of integrating functional MR imaging with radiation therapy treatment by using fiducial gold markers. Radiology. 2005;236(1):311-7.

9. Johnstone E, Wyatt JJ, Henry AM, Short SC, Sebag-Montefiore D, Murray L, et al. Systematic Review of Synthetic Computed Tomography Generation Methodologies for Use in Magnetic Resonance Imaging-Only Radiation Therapy. Int J Radiat Oncol Biol Phys [Internet]. 2018;100(1):199-217. Available from: https://doi.org/https://doi.org/10.1016/j.jijobp.2017.08.043

10. Jonsson $\mathrm{JH}$, Garpebring A, Karlsson MG, Nyholm T. Internal fiducial markers and susceptibility effects in MRI - Simulation and measurement of spatial accuracy. Int J Radiat Oncol Biol Phys. 2012;82(5):1612-8.
11. Van Herk M. Errors and margins in radiotherapy. Semin Radiat Oncol. 2004;14(1):52-64.

12. Owrangi AM, Greer PB, Glide-Hurst CK. MRl-only treatment planning: Benefits and challenges. Phys Med Biol. 2018;63(5):1-30.

13. Nyholm T, Nyberg M, Karlsson MG, Karlsson M. Systematisation of spatial uncertainties for comparison between a MR and a CT-based radiotherapy workflow for prostate treatments. Radiat Oncol. 2009:4(1).

14. Tenhunen M, Korhonen J, Kapanen M, Seppälä T, Koivula L, Collan J, et al. MRI-only based radiation therapy of prostate cancer: workflow and early clinical experience. Acta Oncol (Madr) [Internet]. 2018;57(7):902-7. Available from: https://doi.org/https://doi.org/10.1080/0284186X.2018.1445284

15. Yildirim BA, Onal C, Dolek Y. Is it essential to use fiducial markers during cone-beam CT-based radiotherapy for prostate cancer patients? Jpn J Radiol. 2017;35(1):3-9.

16. Fuller CD, Scarbrough TJ. Fiducial Markers in Image-guided Radiotherapy of the Prostate. Oncol Hematol Rev. 2006;00(02):75.

17. O'neill AGM, Jain S, Hounsell AR, O'sullivan JM. Fiducial marker guided prostate radiotherapy: A review. Br J Radiol. 2016;89(1068):1-18.

18. Deegan T, Owen R, Holt T, Fielding A, Biggs J, Parfitt M, et al. Assessment of cone beam $C T$ registration for prostate radiation therapy: Fiducial marker and soft tissue methods. J Med Imaging Radiat Oncol. 2015;59(1):91-8.

19. Alexander SE, Kinsella J, McNair HA, Tree AC. National survey of fiducial marker insertion for prostate image guided radiotherapy. Radiography [Internet]. 2018;24(4):275-82. https://doi.org/10.1016/j.radi.2018.06.003

20. Maspero M, Van Den Berg CAT, Zijlstra F, Sikkes GG, De Boer HCJ, Meijer GJ, et al. Evaluation of an automatic MR-based gold fiducial marker localisation method for MR-only prostate radiotherapy. Phys Med Biol. 2017;62(20):7981-8002.

21. van der Heide UA, Kotte ANTJ, Dehnad H, Hofman P, Lagenijk JJW, van Vulpen M. Analysis of fiducial marker-based position verification in the external beam radiotherapy of patients with prostate cancer. Radiother Oncol. 2007;82(1):38-45.

22. Kupelian PA, Langen KM, Willoughby TR, Zeidan OA, Meeks SL. ImageGuided Radiotherapy for Localized Prostate Cancer: Treating a Moving Target. Semin Radiat Oncol. 2008;18(1):58-66.

23. Ghadjar P, Fiorino C, Munck af Rosenschöld P, Pinkawa M, Zilli T, van der Heide UA. ESTRO ACROP consensus guideline on the use of image guided radiation therapy for localized prostate cancer. Radiother Oncol [Internet]. 2019;141:5-13. https://doi.org/10.1016/j.radonc.2019.08.027

24. Gustafsson C, Korhonen J, Persson E, Gunnlaugsson A, Nyholm T, Olsson LE. Registration free automatic identification of gold fiducial markers in MRI target delineation images for prostate radiotherapy: Med Phys. 2017:44(11):5563-74.

25. Tyagi N, Fontenla S, Zhang J, Cloutier M, Kadbi M, Mechalakos J, et al. Dosimetric and workflow evaluation of first commercial synthetic CT software for clinical use in pelvis. Phys Med Biol. 2017;62(8):2961-75.

26. Christiansen RL, Jensen HR, Brink C. Magnetic resonance only workflow and validation of dose calculations for radiotherapy of prostate cancer. Acta Oncol (Madr). 2017;56(6):787-91.

27. Bird D, Henry AM, Sebag-Montefiore D, Buckley DL, Al-Qaisieh B, Speight R. A Systematic Review of the Clinical Implementation of Pelvic Magnetic Resonance Imaging-Only Planning for External Beam Radiation Therapy. Int J Radiat Oncol Biol Phys [Internet]. 2019;105(3):479-92. https://doi.org/10. 1016/j.jijobp.2019.06.2530

28. EAU-ESTRO Guideline prostate cancer [Internet]. 2016. Available from: http:// uroweb.org/wpcontent/uploads/EAUGuidelinesProstate-Cancer-2016.pdf

29. Hong CG, Yoon BI, Choe HS, Ha US, Sohn DW, Cho YH. The prevalence and characteristic differences in prostatic calcification between health promotion center and urology department outpatients. Korean $\mathrm{J}$ Urol. 2012;53(5):330-4.

30. Ullrich T, Quentin M, Oelers C, Dietzel F, Sawicki LM, Arsov C, et al. Magnetic resonance imaging of the prostate at 1.5 versus $3.0 \mathrm{~T}$ : A prospective comparison study of image quality. Vol. 90, European Journal of Radiology. 2017. p. 192-7.

\section{Publisher's Note}

Springer Nature remains neutral with regard to jurisdictional claims in published maps and institutional affiliations. 